\title{
樆 \\ First report of strawberry crinivirus 3 and strawberry crinivirus 4 on strawberry in China
}

\author{
D. Chen ${ }^{1}$, X. Ding ${ }^{1}$, A. Wang ${ }^{2}$, J. Zhang ${ }^{1}$ and Z. Wu ${ }^{1}$ \\ ${ }^{1}$ Key Laboratory of Plant Virology of Fujian Province, Institute of Plant Virology, Fujian Agriculture and Forestry University, \\ Fuzhou, Fujian, 350002, China; ${ }^{2}$ London Research and Development Centre, Agriculture and Agri-Food Canada, London, \\ Ontario, N5V 4T3, Canada
}

*E-mail: dingxinlun@163.com

Received: 17 Jan 2018. Published: 21 Jun 2018.

Strawberry (Fragaria $\times$ ananassa) is grown in almost all regions of China. As a result of the rapid expansion in cultivation in recent years, China has become the world's largest strawberry producer (Wu et al., 2009). In the past several years, viral diseases have emerged as a serious threat to strawberry production in many countries.

To monitor the viral pathogen(s) on strawberry in Fuzhou, Fujian province, China, strawberry plants showing virus-like symptoms were collected from commercial fields in April and May 2016. Small RNAs from 12 strawberry plant samples were isolated and mixed into two pools of sRNAs, each pool representing six plants each. The NEBNext Multiplex Small RNA Library Prep Set was used for sRNA library construction and next-generation sequencing (NGS) was done using a paired-end $(2 \times 150)$ configuration on an Illumina MiSeq. NGS reads were trimmed with Trimmomatic (Bolger $e t$ $a l ., 2014)$ and assembled with Velvet (EMBL-EBI) and SeqMan software (Lasergene). BlastN (Altschul et al., 1990) was used to compare the resulting contigs against the NCBI database. Eleven contigs (ranging from 59 to $293 \mathrm{nt}$ ) obtained by NGS were found to share $98-100 \%$ nucleotide sequence identity with the partial genomic sequence of the putative strawberry crinivirus 3 (SCrV-3) isolate M1 (GenBank Accession No EU267168) from Maryland, USA. Another nine contigs (ranging from 53 to $115 \mathrm{nt}$ ) shared $97-100 \%$ nucleotide sequence identity with the partial genomic sequence of a putative strawberry crinivirus 4 ( $\mathrm{SCrV}-4)$ isolate B1156-M3 (EU490423) also from Maryland, USA. Strawberry vein banding virus, which is already known to infect strawberry in China, was also identified by Blast searches.

RT-PCR was used to verify the presence of SCrV-3 and SCrV-4 in the strawberry plants. RT-PCR primers, SCrV3f1 (5'-AGCTTCGTCGCGTTAACGTGGAC-3') and SCrV3r1 (5'-GATTTCGCCTTCACAACGTCATAGTG-3'), were designed from the sequence of a ScrV3 isolate (EU267168) and generated a $1775 \mathrm{bp}$ amplicon from two samples. The cloned and sequenced amplicon shared 99\% nucleotide identity with the US SCrV-3 isolate (EU267168), confirming that the virus discovered in this study is SCrV-3. The Chinese isolate was named SCrV-3 FZ (KX852314). RT-PCR primers, SCrV4f1 (5'-AATTCTGATCCTATCCTTAGT-3')

and SCrV4r1(5'-TGGTGATTGTGCTACTTCTTTAGC-3'), were designed from the sequence of a ScrV4 isolate (EU490423) and generated a $1452 \mathrm{bp}$ amplicon from three samples. The cloned and sequenced amplicons shared 99\% nucleotide identity with the American SCrV-4 isolate, and an isolate (KU237245) recently found in Canada (Ding et al., 2016). The Chinese isolate was named SCrV-4 FZ1 (KY488557).

A further 90 strawberry plants with virus-like symptoms collected from other regions in Fujian province were tested by RT-PCR for both viruses. $\mathrm{SCrV}-3$ and SCrV-4 were not detected in any sample, suggesting that these viral pathogens are not widespread in this province. To our knowledge, this is the first report of SCrV-3 and SCrV-4 on strawberry in China. Further work is needed to determine the occurrence and distribution of $\mathrm{SCrV}-3$ and SCrV-4, and its impact on strawberry production in China.

\section{References}

Altschul SF, Gish W, Miller W, Myers EW, Lipman DJ, 1990. Basic local alignment search tool. Journal of Molecular Biology 215, 403-410. http://dx.doi.org/10.1016/S0022-2836(05)80360-2

Bolger AM, Lohse M, Usadel B, 2014. Trimmomatic: a flexible trimmer for Illumina sequence data. Bioinformatics 30, 2114-2120. http://dx.doi.org/10.1093/bioinformatics/btu170

Ding XL, Li YZ, Hernández-Sebastià C, Abbasi PA, Fisher P, Celetti MJ, Wang AM, 2016. First report of strawberry crinivirus 4 on strawberry in Canada. Plant Disease, 1254.

http://dx.doi.org/10.1094/PDIS-01-16-0009-PDN

Wu YJ, Li YS, Zhao YH, Wu YQ, Cheng HH, 2009. Application status and development of biotechnology in strawberry production. Journal of Hebei Agricultural Sciences 13, 41-44.

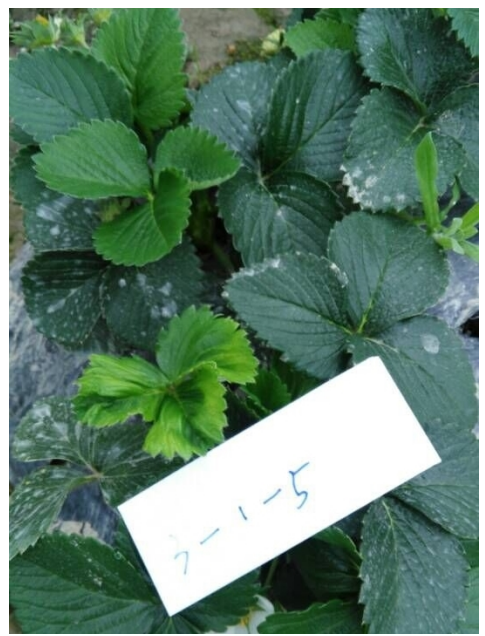

Figure 1

To cite this report: Chen D, Ding X, Wang A, Zhang J, Wu Z, 2018. First report of strawberry crinivirus 3 and strawberry crinivirus 4 on strawberry in China. New Disease Reports 37, 24. http://dx.doi.org/10.5197/j.2044-0588.2018.037.024

(c) 2018 The Authors

This report was published on-line at www.ndrs.org.uk where high quality versions of the figures can be found. 